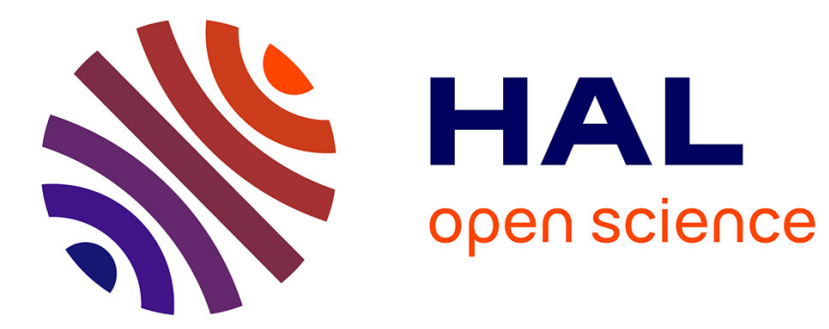

\title{
Les besoins socio-cognitifs fondamentaux du jeune enfant face à l'écran
}

Emmanuel Devouche

\section{To cite this version:}

Emmanuel Devouche. Les besoins socio-cognitifs fondamentaux du jeune enfant face à l'écran. Les Métiers de la petite enfance, 2019, 25, pp.14 - 16. 10.1016/j.melaen.2019.01.003 . hal-03487965

\section{HAL Id: hal-03487965 https://hal.science/hal-03487965}

Submitted on 20 Dec 2021

HAL is a multi-disciplinary open access archive for the deposit and dissemination of scientific research documents, whether they are published or not. The documents may come from teaching and research institutions in France or abroad, or from public or private research centers.
L'archive ouverte pluridisciplinaire HAL, est destinée au dépôt et à la diffusion de documents scientifiques de niveau recherche, publiés ou non, émanant des établissements d'enseignement et de recherche français ou étrangers, des laboratoires publics ou privés.

\section{다)(1) $(5$}

Distributed under a Creative Commons Attribution - NonCommercial| 4.0 International 
Dochead dossier

Sous-dochead L'impact des écrans sur la relation avec le tout-petit

développement

\title{
Les besoins psycho-cognitifs fondamentaux du jeune enfant face à l'écran
}

\author{
Emmanuel Devouche ${ }^{a, b}$ \\ $\mathrm{PhD}$, enseignant chercheur \\ a Université Paris-Descartes, Laboratoire de psychopathologie et processus de santé (EA 4057), \\ 92774 Boulogne-Billancourt cedex, France \\ ${ }^{b}$ EPS Erasme, Laboratoire RePPEr, 14 rue de l'Abbaye, 92160, Antony, France \\ Adresse e-mail : devouche7@gmail.com (E. Devouche).
}

Résumé

Les écrans sont omniprésents dans notre quotidien. Leur usage par les jeunes enfants, en particulier s'il n'est pas accompagné, nuit à leur développement psycho-cognitif, affectif et social. En effet, comme tout être humain, le petit enfant est un être de relation. Le rôle de l'adulte est de réguler I'usage des écrans pour privilégier une relation authentique et sécure avec les tout-petits.

(C) 2019

Mots clés - accompagnement ; communication ; développement ; écran ; régulation ; relation

Au cours des dernières décennies, nous avons beaucoup appris des recherches réalisées durant la petite enfance. Nous savons à présent que le bébé vient au monde avec de nombreuses compétences, qui trouvent leurs racines durant la période anténatale. II naît avec une appétence particulière pour le contact humain, ce qui est emblématique de ce que nous sommes au commencement: des êtres sociaux qui s'épanouissent dans l'interaction avec l'autre. L'écran (télévision, tablette, téléphone) arrive alors comme un anachronisme, ne répondant pas à ses besoins fondamentaux, et menaçant d'appauvrir, voire d'entraver, son développement.

\section{T1 Un développement socio-cognitif fulgurant}

TEG1 Très précocement, le bébé possède les compétences nécessaires pour entrer en communication avec les individus de son environnement, ses parents, ses frères et sœurs, etc. Tout au long de la petite enfance, il développe cette compétence à interagir, à être avec, à comprendre les autres, motivé par la curiosité et le désir d'être complice. En l'espace d'une année, ses capacités à communiquer, son intelligence sociale vont se développer de manière spectaculaire.

TEG1 Autour de 2-3 mois, les scientifiques s'accordent à dire que le bébé est capable de s'engager dans un échange conversationnel et son sourire davantage contrôlé prend un caractère évidemment social. Autour de 6 mois, alors que s'amorce la maîtrise de la posture assise, il commence à partager son attention avec un partenaire, sur un jeu ou un livre, par exemple. Au cours des semaines qui suivent s'observent les prémices de la compréhension des émotions d'autrui. Cette capacité appelée 
référenciation sociale lui permet d'appréhender une situation en fonction de l'expression d'autrui, enrichissant sa façon de communiquer et, surtout, lui permettant d'explorer l'environnement tout en gardant un contact avec le partenaire. Nous voyons aussi émerger durant cette période de la vie des émotions comme la timidité, la taquinerie, l'espièglerie, en bref, la capacité non seulement à comprendre les émotions de l'autre mais également à jouer avec, le bébé aimant par exemple reproduire une action qui fait rire.

TEG1 À la fin de la première année, le bébé devient peu à peu capable d'attention conjointe, autrement dit non seulement celle d'être avec, mais de faire avec, une capacité qui va de pair avec l'essor du babillage et l'autonomie du déplacement. Cette compétence marque un tournant dans son développement car elle permet à l'enfant d'être davantage acteur et complice dans son exploration du monde.

TEG1 Au cours de la deuxième année de la vie, toutes ces acquisitions se solidifient, se diversifient, et de nouvelles apparaissent : sur le plan posturo-moteur, telles la marche ou la manipulation fine, permettant par exemple l'utilisation de la cuillère ; sur le plan de la socialisation, notamment avec l'apprentissage des conventions, et sur le plan du langage qui explose à la fin de la deuxième année. Ce développement fulgurant se poursuit tout au long de la petite enfance et de l'enfance. Toutefois, si chaque enfant est unique et se développe à son propre rythme, tous ont en commun un besoin d'être accompagné et régulé, car ce développement ne peut se faire seul.

\section{T1 Accompagné et régulé pour mieux se développer}

TEG1 Les adultes qui composent l'environnement social du jeune enfant (parents, professionnels des lieux d'accueil, etc., les "donneurs de soins") jouent un rôle essentiel pour accompagner le développement, et ce, bien au-delà de la petite enfance d'ailleurs. Soutien, étayage, enveloppe, aide, accompagnement, régulation, le rôle de l'entourage peut être désigné de différentes façons qui, toutes, expriment la nécessité d'être ajusté à chaque instant. Lui répondre ou intervenir au bon moment, lui laisser de l'espace de parole ou le laisser faire seul pour le laisser découvrir par luimême, être ajusté, prennent des formes subtiles qui s'acquièrent au fil du temps, au fur et à mesure de la complicité qui s'installe.

TEG1 Chaque enfant est unique dans ses besoins d'accompagnement et de régulation. Certains ont dès la naissance des difficultés à s'autoréguler, à gérer un environnement surstimulant, tandis que d'autres paraissent appréhender le monde avec davantage de facilité. Pour tous les enfants, communiquer avec l'autre, explorer le monde demandent des ressources et une certaine disponibilité (ne pas avoir faim, ne pas être fatigué, etc.). Ces ressources et cette disponibilité varient d'un enfant à l'autre, et d'un jour à l'autre chez un même enfant, rendant difficile le rôle de l'adulte donneur de soins.

TEG1 Pour l'adulte qui prend soin de l'enfant, il s'agit d'être sensible et ajusté - responsive en anglais -, autrement dit, répondre de manière adéquate et cohérente. Et il s'agit, aussi et surtout, de répondre dans la bonne temporalité, au bon moment. L'adulte doit alors se mettre au niveau de l'enfant, doit comprendre ses besoins pour s'y ajuster. Pour cela, il doit aussi mobiliser ses ressources et être disponible.

TEG1 Sans l'adulte présent au quotidien pour envelopper son activité, pour l'accompagner, pour décoder et comprendre le monde qui l'entoure, le jeune enfant se trouve livré à lui-même. L'environnement dans lequel il évolue n'est pas toujours adapté, et il doit faire face quotidiennement à des situations nouvelles. Il y parviendra parfois, mais aura souvent besoin d'autrui pour cela car le monde prend sens au travers de cette activité partagée. Et c'est au travers de cette activité partagée que le jeune enfant se dépasse, se développe et grandit (encadré 1). 


\section{T1 À l'ère du numérique, le bébé face à l'écran}

Comme la lecture d'un livre pour enfants gagne à être accompagnée d'un adulte, en va de même avec un écran, qu'il s'agisse de la télévision, d'une tablette, d'un ordinateur ou d'un téléphone. Comment le jeune enfant peut-il comprendre ce qu'il y voit si nous ne sommes pas là pour l'y aider, en verbalisant, en rassurant, en expliquant ? L'écran sous toutes ses formes fait désormais partie du quotidien, alors comment faire?

Pour répondre à cette question, il faut prendre conscience et garder à l'esprit que l'usage d'un écran comprend plusieurs spécificités :

- l'image se met à bouger et à parler toute seule, sans que le jeune enfant puisse la contrôler, risquant d'entraver et d'appauvrir son imaginaire ;

- les objets s'animent mais ne peuvent plus être attrapés ou manipulés parce qu'ils n'existent pas vraiment, risquant d'entraver l'exploration du jeune enfant et d'appauvrir peu à peu sa compréhension du monde ;

- les images défilent sans considération pour la temporalité du jeune enfant, risquant d'entraver sa possibilité d'être acteur et son désir de partage, et d'appauvrir sa capacité à interagir ;

- l'écran ne communique pas avec le jeune enfant, ne s'ajuste pas à lui, ne l'enveloppe pas, ne lui répond pas ;

- l'écran est qualifié d'interactif... mais il n'est pas une personne ;

- seul face à l'écran, le jeune enfant n'est plus acteur, il n'est plus complice ;

- seul face à l'écran, le jeune enfant ne peut plus faire l'effort de comprendre, se dépasser, se développer et grandir.

\section{T1 Conclusion}

L'écran est inutile, voire nocif, au développement du très jeune enfant. Son usage fait désormais partie du quotidien mais il n'est pas une fatalité, même à l'ère du numérique : l'écran peut rester éteint la plupart du temps. Nous pouvons passer du temps avec nos enfants en laissant notre téléphone de côté : en effet, l'écran occupe la place que nous lui donnons, au même titre que les livres ou que n'importe quel jouet. En parallèle, l'écran peut être un formidable moyen de parler à distance aux grands-parents, de diffuser de la musique, de regarder des films autour desquels les membres de la famille peuvent échanger ensuite. En somme, l'accès aux écrans peut être proposé avec modération, et il doit être accompagné, au même titre que toute activité.

\section{Déclaration de liens d'intérêts}

L'auteur déclare ne pas avoir de liens d'intérêts.

\section{Références}

Références à appeler tout au long du texte (en attente).

\section{Pour en savoir plus}

Fondation pour l'enfance. Journée nationale d'informations et de repères sur le numérique des toutpetits. Paris, 5 novembre 2018.

www.fondation-enfance.org/2018/11/08/journee-nationale-dinformations-de-reperes-numeriquepetits-5-novembre-2018/ 
Encadré 1. La lecture, une activité partagée pour un développement accompagné.

Le jeune enfant peut manipuler seul et sans danger un livre qui lui est destiné, regarder les images qu'il contient et y prendre du plaisir. En le regardant avec lui, l'adulte lui donne un sens différent en mettant les images en mots, en faisant intervenir des émotions avec la voix, avec l'expression du visage ou avec le corps. Il fait vivre ce livre en l'associant à du plaisir partagé. Et quand ce livre est partagé avec papa ou avec maman, cela donne encore plus de richesse, l'un et l'autre vont le lire différemment, alimentant l'imaginaire et la complicité.

Parfois, dans les histoires pour enfants, il se trouve un loup, ou une image nouvelle qui peut paraître bizarre. Quelquefois les images paraissent anodines à l'adulte mais le jeune enfant les perçoit autrement, car il les voit avec ses yeux d'enfant. Quel imaginaire développe-t-il s'il est seul face à ces images ? Comment peut-il les comprendre si nous ne sommes pas là pour l'y aider, en verbalisant, en rassurant, en expliquant, en rendant le loup gentil, et l'image bizarre, rigolote ?

\section{Illust1-Devouche.jpg}

\section{(C) Voisin/Phanie}

Légende : Comme la lecture d'un livre pour enfants gagne à être accompagnée d'un adulte, il en va de même avec un écran 